\title{
Musculoskeletal analysis of driving fatigue: The influence of seat adjustments
}

\author{
Noor Aliah binti Abdul Majid ${ }^{1,}$, Mohd Fareez Edzuan Abdullah ${ }^{1, b}$, \\ Mohd Syahmi Jamaludin ${ }^{1, c}$, Mitsuo Notomi ${ }^{2, d}$ and John Rasmussen ${ }^{3, \text { e }}$ \\ ${ }^{1}$ Department of Mechanical and Manufacturing Engineering, Universiti Malaysia Sarawak, 94300 \\ Kota Samarahan, Sarawak, Malaysia \\ ${ }^{2}$ Department of Mechanical Engineering, Meiji University, 214-8571, Japan \\ ${ }^{3}$ Department of Mechanical and Manufacturing Engineering, Aalborg University, Denmark \\ an.aliah.abdmajid@gmail.com, bamfareez@feng.unimas.my, cjmsyahmi@feng.unimas.my, \\ dnotomim@isc.meiji.ac.jp, ejr@me.aau.dk
}

Keywords: inverse dynamics analysis, musculoskeletal model, muscle activity, intradiscal force

\begin{abstract}
Main causes for discomfort experienced by vehicle drivers during driving were investigated using a rigid-body model originally developed in the AnyBody Modeling System [1]. The interactions between the human body and the car-seat in various combinations of seat-pan/backrest inclinations and the effect of pedal spring stiffness were analyzed using an inverse dynamics approach. To deal with the muscle redundancy problem, (i.e. the problem with the human-body containing more muscles than necessary to drive its degrees of freedom) a "minimum-fatigue" criterion [2] was utilized. The results show that various seat adjustments (e.g., seat-pan and backrest inclinations) and the pedal spring stiffness have complex influences on the muscle activation and spinal joint forces of the human body. From the results, an optimal adjustment for the car-seat is proposed, i.e. the backrest inclination is $10^{\circ}$ and the seat-pan inclination is between $0^{\circ}$ to $5^{\circ}$. This study can in general capture the overall interactions between human body and environment (i.e. the maximum muscle activity and spine forces), which is thought to be the factors of driving fatigue.
\end{abstract}

\section{Introduction}

The use of computer models of humans and seats for analyzing their interactions can speed up and economize the process of development and introduction of new, more comfortable car-seats. In earlier stages of seat designing process, new designs can be tested for its degree of comfort by carrying out computer simulations of the human interactions with the seat. To address some of the limitations of the frequently cited analyses, which are mostly based on the measurements of the distribution of human/seat contact pressure over the contact area (example [3] to [5]), seated-human and car-seat interactions associated with typical sitting postures of the vehicle driver are analyzed using an inverse dynamic approach. In this paper, a musculoskeletal simulator, namely the Anybody Modeling System [1] and its associated public-domain library of body models are being used and further developed to examine the influence of different car seat adjustments and the accelerator pedal's spring stiffness on muscular activity and spinal joint forces during the act of driving.

\section{Method}

The AnyBody Modeling System. The AnyBody Modeling System [1] developed at Aalborg University and used in the present work is a musculoskeletal modeling and simulation program; described in detail by Damsgaard et al. [6]. The system can model the musculoskeletal system and the environment that interacts with human body; compute forces in individual muscles, elastic energy in tendons, joint reactions etc. Each body model consists of segments (bones), joints between segments and tendon-muscle units. 
In the system, a typical musculoskeletal multi-body dynamics problem is solved using inverse dynamics methods, within which the desired "known" motion (e.g. posture, velocity, acceleration) and external load are prescribed and the "unknown" muscle activity required to produce this motion is computed. The redundancy problem (i.e. human body contains more muscles than what would be typically needed to drive various body joints) is solved using an optimization-based approach [6]. The mathematical form of the inverse dynamic problem can be stated as follow:

Minimize the objective function:

$$
G\left(f^{(\mathrm{M})}\right)
$$

Subjected to the following constraints:

$$
\begin{aligned}
& \mathbf{C f}=\mathbf{d} . \\
& f_{i}^{(\mathrm{M})} \geq 0, i \in\left\{1, \ldots, n^{(\mathrm{M})}\right\} .
\end{aligned}
$$

where $G$ in Eq. (1) is the objective function of the recruitment strategy stated in terms of the muscle forces $f_{i}^{(\mathrm{M})}$, and minimized with respect to all unknown forces in the problem, $\mathbf{f}$ (i.e. muscle forces and joint reactions). Eq. (2) is the dynamic equilibrium equation, where $\mathbf{C}$ is the coefficient matrix for the unknown forces/moments in the system while $\mathbf{d}$ is a vector of the known applied loads and inertia forces. The non-negativity constraints on the muscle forces, Eq. (3), states that muscle can only pull, not push. Although there are many forms of objective functions, $G$ (refer [2]), the most reasonable criteria are functions of the normalized muscle forces, $f_{i}^{(\mathrm{M})} / N_{i}$, where $N_{i}$ is some measure of the muscle strength at each muscle's current working conditions. In this work, the following form is adopted:

$$
\text { Minimize } \max \left(\frac{f_{i}^{(\mathrm{M})}}{N_{i}}\right) \text {. }
$$

The min/max muscle recruitment formulation (Eqs. (2) (4)), is effectively a minimum fatigue criterion, i.e. the muscles are recruited to postpone fatigue of the highest relatively loaded muscle as far as possible, and under the assumption that the Central Nervous System (CNS) in some sense tries to minimize the load on the muscles and the body in general. Particularly, in the muscle groups associated with the maximum muscle activity, there will be many muscles which, in a coordinated manner, carry a portion of the load comparable with their individual strengths. Consequently, a distinctive envelope of muscles with the same activity level, which called "the muscle activity envelope", is formed. The muscle activity envelope appears to be an important parameter for ergonomic design optimization; designs that are associated with lower envelope levels may be perceived as less fatigue-inducing.

Musculoskeletal Model. The musculoskeletal model of the human body used in this research was downloaded from a public-domain AnyScript Model Repository version 1.2 [7], namely "the seated human model" (Fig. 1); constructed and described in detail by Damsgaard et al. [6]. A detailed description of taxonomy of the human body model used in the present work was reviewed by Rasmussen et al. [8], and is therefore omitted in this paper. Muscles are modeled without contraction dynamics (i.e. the strength of the muscle model is independent of the muscle's current length and contraction velocity). It is known that the muscles do not behave that way, but since the present problem involves moderate contraction velocities and small joint angle variations, this approximation is acceptable. 
Car-Seat Model. The rigid-body car-seat model comprises of head-rest, backrest, seat-pan and foot-rest. Kinematic links were placed at the human-body/seat interface to obtain desired posture of the human for a given adjusted configuration of the seat. The links were not allowed to transmit any forces/moments; instead, the human-body/car-seat contact forces have been accounted for through the use of "support points" shown in Fig. 2, which was described in detail by Rasmussen et al. [8].

Analysis Condition. To analyze driving fatigue sensed by automobile drivers, the human-body model was first placed in the generic car-seat. Two additional segments were added to represent the brake pedal/ accelerator assembly and a steering wheel. Then, the human-body was positioned in accordance with a typical posture associated with vehicle driving, involving positioning the right foot on the accelerator pedal while left foot resting on the footrest and both hands were fixed at the steering wheel. First, to analyze the pedal pressing operation, the pedal is driven to $30^{\circ}$ at constant acceleration. Then, to investigate the steering wheel turning operation, the steering wheel is turned from initial position $\left(0^{\circ}\right)$ to $90^{\circ}$ clockwise. In both cases, the seat was adjusted according to the parameters shown in Table 1, which is called the "reference case" parameters.

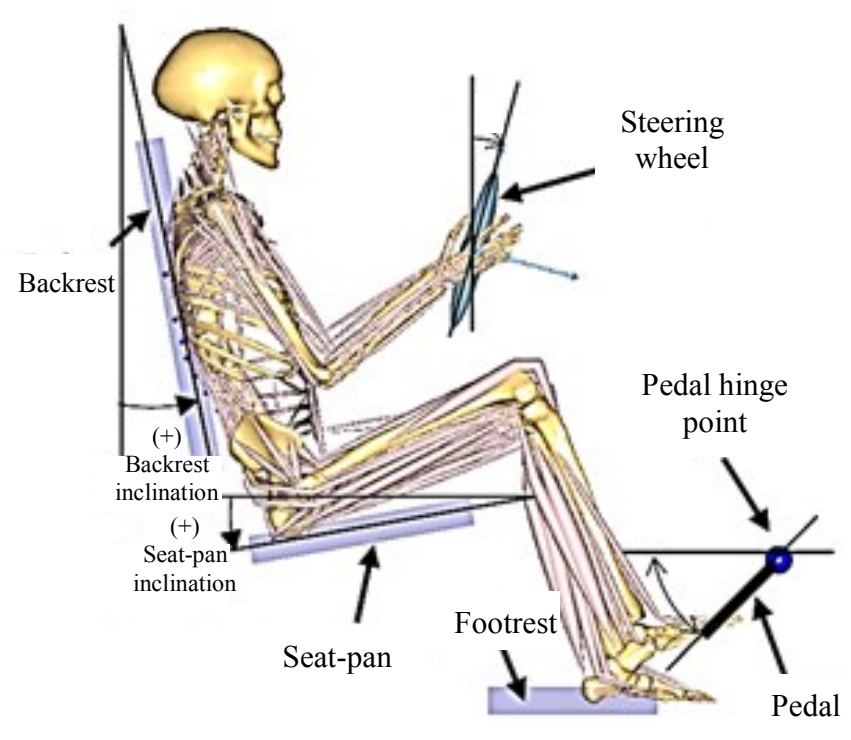

Fig. 1 The "seated human" model with steering wheel and accelerator pedal.

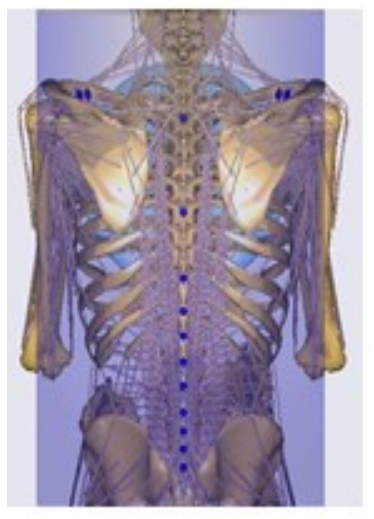

(a)

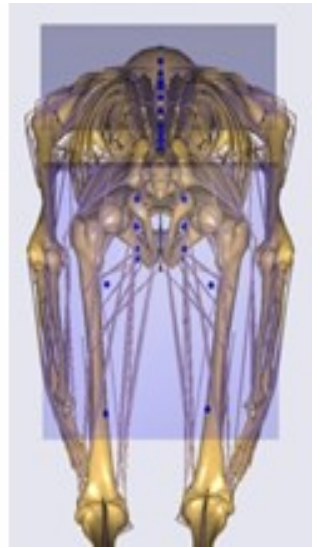

(b)
Fig. 2 Support points (blue spheres) in the model through the partially transparent seat: (a) posterior view on the spine and shoulder and (b) inferior view under the thighs and pelvis.

Table 1 Reference case parameters

\begin{tabular}{l|l} 
Parameter & Value \\
\hline \hline Backrest inclination & $10^{\circ}$ \\
\hline Seat-pan inclination & $10^{\circ}$ \\
\hline Accelerator pedal spring stiffness & $25[\mathrm{Nm} / \mathrm{rad}]$ \\
\hline Steering wheel torque & Maximum 5 $[\mathrm{Nm}]$ \\
\hline
\end{tabular}

\section{Results}

The effect of seat-pan and backrest inclination. The influence of seat-pan and backrest inclination angles on muscle activity and spinal joint forces was investigated (refer Fig. 3 and Fig. 4). The seat-pan inclination angle ranges from $0^{\circ}$ through $15^{\circ}$ and the backrest inclination angle ranges from $0^{\circ}$ through $30^{\circ}$; both in increments of $5^{\circ}$, totaling of 28 combinations of seat 
adjustments. The highest values of muscle activity over the analyzed motion and highest value of the intradiscal compressive force between the fourth and fifth lumbar vertebrae (L4-L5) of each seat configuration during the pedal pressing operation are plotted in the surface graph (Fig.3). From Fig. 3 (a), the muscle activity is relatively lower when the backrest is inclined backward. Although the maximum muscle activity can be decreased approximately $2 \%$ by inclining the backrest, it has the tendency to increase slightly when the seat-pan is inclined. As can be seen in Fig. 3 (b) the intradiscal compressive force increases when backrest is inclined.

From Fig. 3, it can be seen that the backrest inclination has more influence on the body than the seat-pan does during the pedal pressing operation. Therefore, the effect of backrest inclination was observed. Fig. 4 (a) shows the average muscle activity envelope in each muscle group (i.e. trunk, right leg, left leg, right shoulder and left shoulder). The result indicates that for the most part, the muscle activation monotonically increases or decreases with the backrest inclination angle. Fig. 4 (b) shows the influence on spinal joint force. From the figure, it can be seen that the force decreases when backrest is inclined until $20^{\circ}$, and then increases when inclined further. However, this result is unexpected since in reality the backrest should support the body, causing lower force on the spine.

Fig. 5 shows the effects of seat adjustments on muscle activity and spine forces during steering wheel turning operation. Fig. 5 (a) shows that while inclining the backrest backward may reduce the muscle activity from $10 \%$ to $9 \%$, inclining the seat-pan does not have a measurable influence on the muscle activity when the driver is turning the steering wheel. The highest value of the intradiscal compressive force between L4-L5vertebrae is presented in Fig. 5(b). The result shows that increasing the backrest inclination angle to $20^{\circ}$ is beneficial to the spine; it reduces the compressive force to approximately $40 \%$ lower than without inclining.

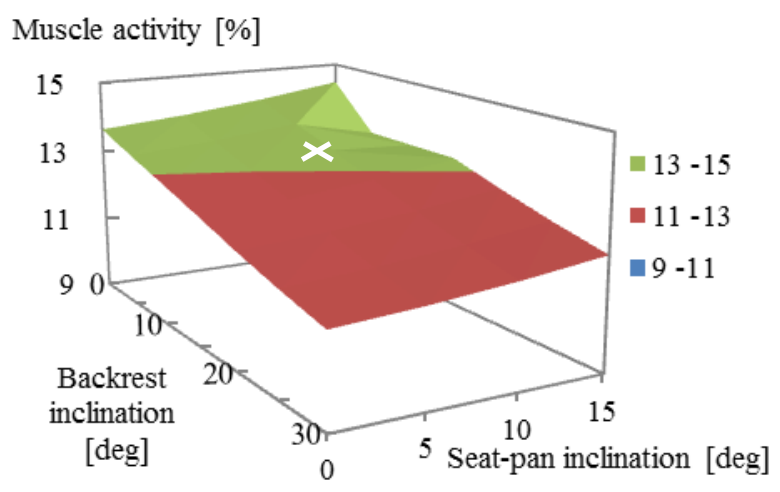

(a)

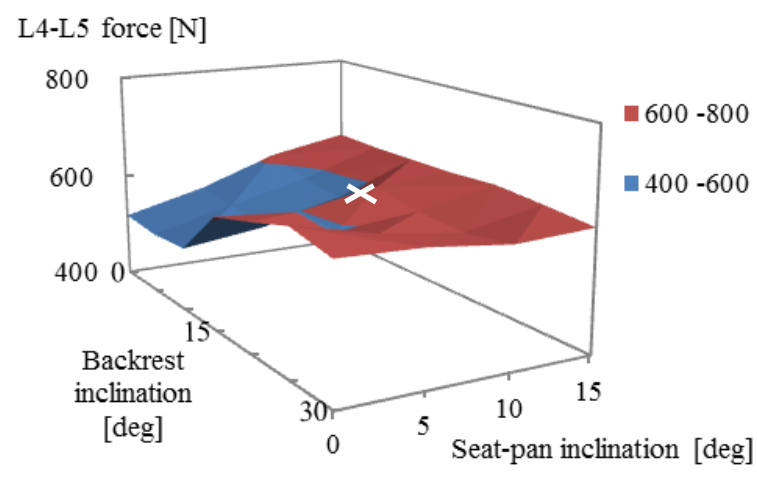

(b)

Fig. 3 Pedal pressing analysis: (a) Maximum muscle activity (b) L4-L5 reaction force as a function of seat-pan and backrest inclination (reference case is marked by white cross).

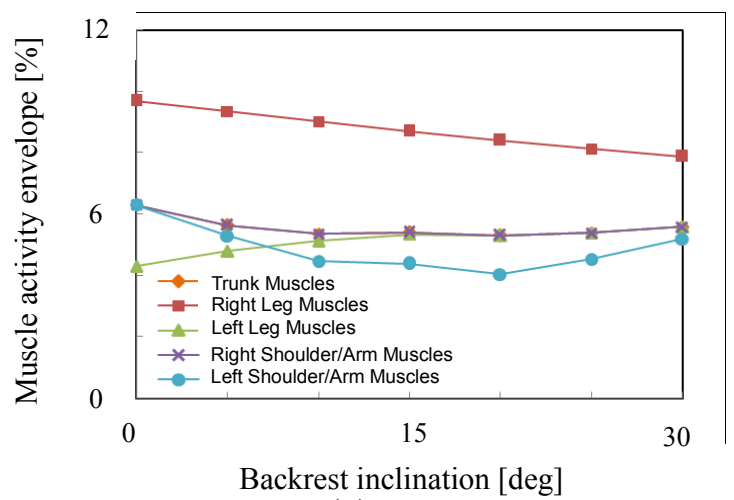

(a)

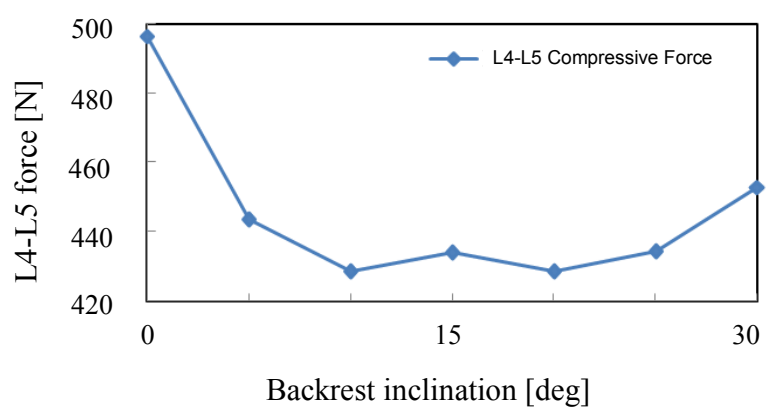

(b)

Fig. 4 The effect of backrest inclination on: (a) Muscle activity envelope (b) L4-L5 intradiscal compressive reaction force. 


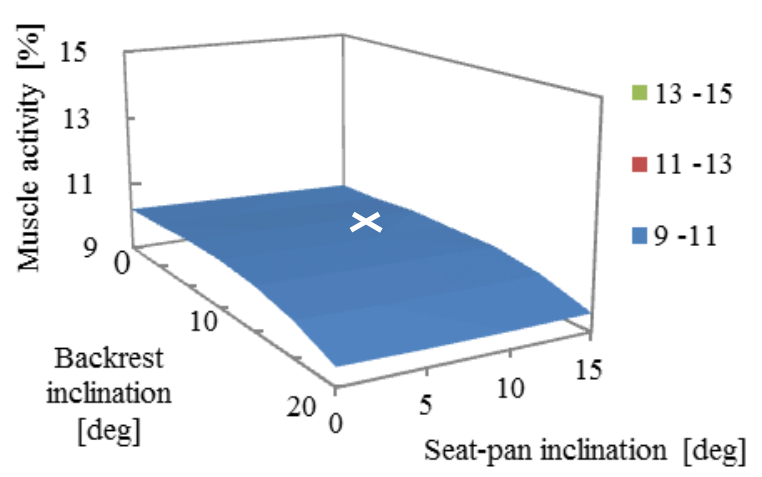

(a)

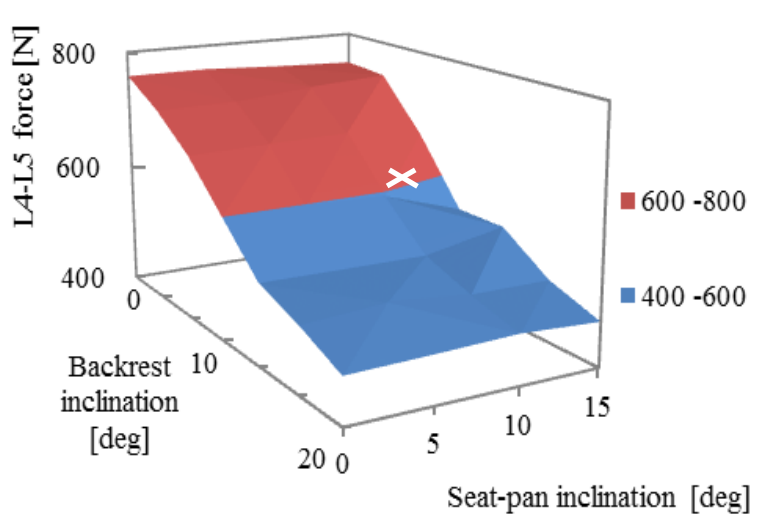

(b)

Fig. 5 Steering wheel turning analysis: (a) Maximum muscle activity (b) L4-L5 reaction force as a function of seat-pan and backrest inclination (reference case is marked by white cross).

The effect of pedal spring stiffness. The influence of accelerator pedal's spring stiffness was observed by placing an external force representing the pedal resistance. The value was set in 5 to 30 $\mathrm{Nm} / \mathrm{rad}$ range in the increments of $5 \mathrm{Nm} / \mathrm{rad}$. Fig. 6 (a) shows the muscle activity envelope as a function of pedal angle from analysis of pedal pressing operation. As can be seen here, in the cases of pedal spring stiffness ranged from $25 \mathrm{Nm} / \mathrm{rad}$ to $30 \mathrm{Nm} / \mathrm{rad}$, the activity increased with the pedal angle. This characteristic is advantageous to the driver to control the pedal since it requires more force to push the pedal even further. The intradiscal compressive force between L4-L5 vertebrae in the pedal pressing analysis is shown in Fig. 6 (b). It is clear from this figure that the spring stiffness of $25 \mathrm{Nm} / \mathrm{rad}$ is beneficial to the driver as the spine force decreases tremendously at the beginning of the motion compared to when the stiffness is lower.

\section{Discussion}

Fig. 7 shows a superimposed figure of the muscle activity envelope and force between L4-L5 vertebrae during pedal pressing operation. The optimal adjustment for backrest inclination is $10^{\circ}$ and seat-pan inclination is between $0^{\circ}$ to $5^{\circ}$. It is thought that the combination of these two parameters value can reduce the fatigue sensed by the driver by lowering the muscle activity and spine forces. This proposed optimal configuration is consistent with the parameters in a review paper by Harrison [9].

\section{Summary}

A model of car-seat/human interface has been designed and their interactions have been investigated in two types of operations: pedal pressing and steering wheel turning. Further work is needed to investigate the distribution of forces over the contact interface and their penetration into human soft tissue. In addition, the effect of vibration should be addressed.

\section{Acknowledgment}

A portion of the material presented in this paper is based on the use of the Anybody Modeling System (Anybody Technology A/S, 2010), a musculoskeletal multi-body dynamics software. The authors are grateful to the Faculty of Engineering, UNIMAS for providing financial support. 


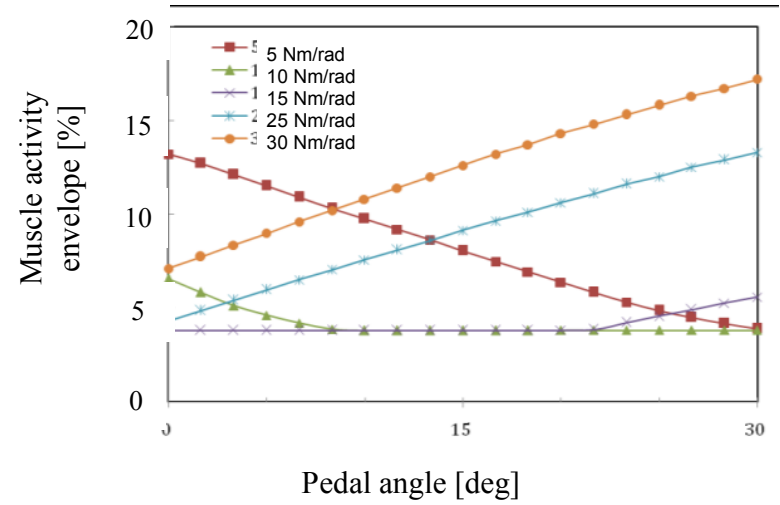

(a)

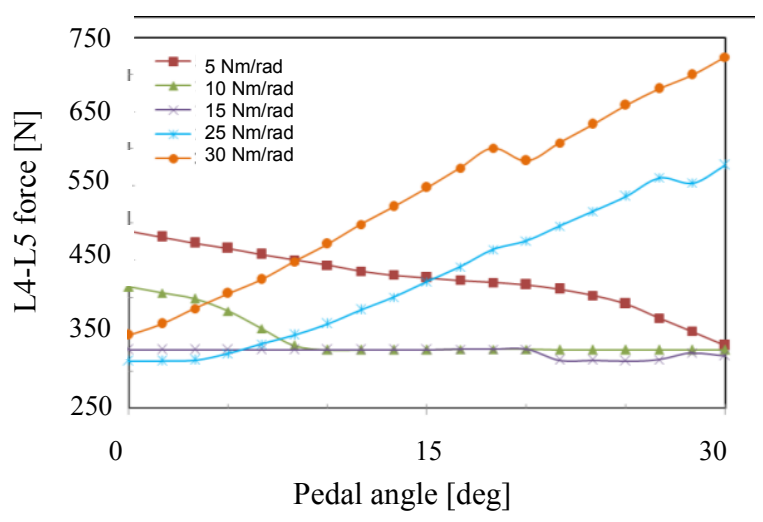

(b)

Fig. 6 The effect of pedal spring stiffness on: (a) Muscle activity envelopes and, (b) Intradiscal L4-L5 compressive reaction force.

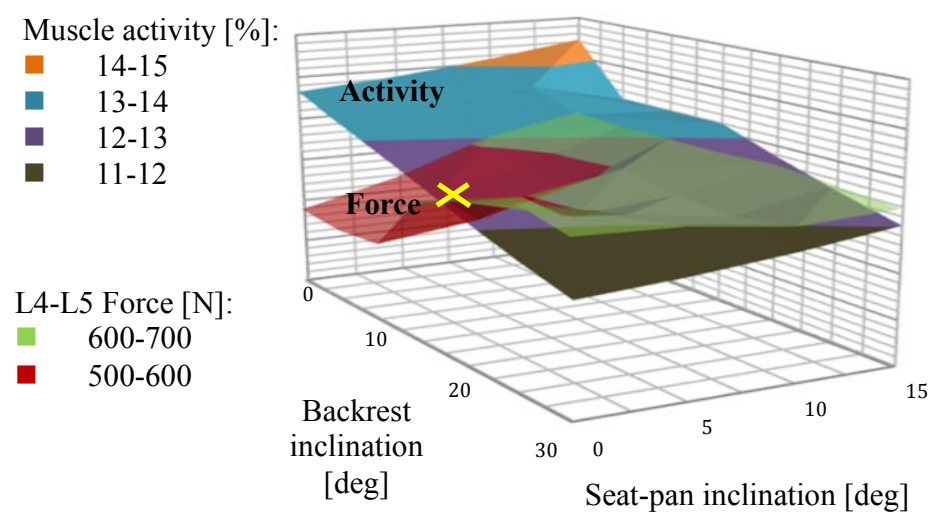

Fig. 7 The superimposed surface graph of muscle activity and spine force (the proposed optimal seat adjustments result is shown by yellow cross).

\section{References}

[1] AnyBody 4.2, AnyBody Technology A/S, Aalborg, Denmark, 2010.

[2] J. Rasmussen, M. Damsgaard, M. Voigt, Muscle Recruitment by the $\mathrm{min} / \mathrm{max}$ Criterion: A Comparative Numerical Study, J. Biomech. 34 (2001) 409-415.

[3] G. Kyung, M. A. Nussbaum, K. Babski-Reevesb, Driver sitting comfort and discomfort (part 1): use of subjective ratings in discriminating car seats and correspondence among ratings, Int. J. Ind. Ergon. 38 (2008) 516- 525.

[4] G. Kyung, M. A. Nussbaum, Driver sitting comfort and discomfort (part II): relationships with and prediction from interface pressure, Int. J. Ergon. 38 (2008) 526-538.

[5] P. K. Nag, S. Pal, S. M. Kotadiya, A. Nag, K. Gosai, Human-seat interface analysis of upper and lower body weight distribution, Int. J. Ind. Ergon. 38 (2008) 539-545.

[6] M. Damsgaard, J. Rasmussen, S. Torholm, E. Surma, M. de Zee, Analysis of musculoskeletal systems in the Anybody Modeling System, Simulation Modelling Practice and Theory 14 (2006) 1100-1111.

[7] AnyScript Model Repository 1.2, AnyBody Technology A/S, Aalborg, Denmark, 2010.

[8] J. Rasmussen, S. Torholm, M. de Zee, Computational analysis of the influence of seat pan inclination and friction on muscle activity and spinal joint forces, Int. J. Ind. Ergon. 39 (2009) $52-57$.

[9] D. D. Harrison, S. O. Harrison, A. C. Croft, D. E. Harrison, S. J. Troyanovih, Sitting biomechanics, Part II: Optimal car driver's seat and optimal driver's spinal model, J.Manipulative Physiol. Ther. 23(1) (2000) 37-47. 